\title{
Specificity of Organoids for Precision Tissue Regeneration: A Review
}

\author{
Uche A Osunkwo ${ }^{1 *}$, Ezeunala Mercy $N^{2}$, Okolo $R U^{3}$, Bassi $P U^{1}$ and Chinedu Okeke \\ ${ }^{1}$ Department of Pharmacology \& Therapeutics, University of Abuja, Abuja, Nigeria
}

${ }^{2}$ Department of Microbiology and Biotechnology, National Institute for Pharmaceutical Research and Development, Abuja, Nigeria

${ }^{3}$ Department of Anatomical Sciences, University of Abuja, Abuja, Nigeria

${ }^{4}$ Department of Hematology \& Blood Transfusion, College of Health Sciences, University of Abuja, Abuja, Nigeria

\begin{abstract}
Summary
An apparently simple method for generating human retinal organoids has been described by [1]. These investigators cultured cell aggregates in free-floating condition after initially scrapping adherent cells. Their results showed that newly elaborated morphologically well defined optic vesicles emerged within 7 days and were identifiable and could be harvested [1].

Organoids arise from stem cells as three-dimensional self-assembled colloid-like objects inside the culture medium (mimicking in vivo micro-environment) and these organoids carry with them the genetic composition (phenotype) of their parent stem cells and organs. In addition, organoids express the genetic identity of their parental organs. This characteristic has made them suitable for use in drug discovery (testing, efficacy and toxicity), modeling of tissue and organ disease and for prophylactic studies. The molecular mechanisms (both enzymatic and signaling) by which organoids are elaborated enable our understanding of the specificity with which precise tissue regeneration by these organoids takes place. Therefore, gene transcription factors and their interactions with organoids become revisited. In this review, we examine reports of studies which have concentrated on precision tissue regeneration (as observed in degenerative disease conditions) and how (mechanistically) organoids have specifically or precisely effected in-situ gene expression for desired regeneration.
\end{abstract}

\section{Keywords}

Organoids, Stem cells, Tissue regeneration, Precision

\section{Introduction}

Do organoids coexist with stem cells in their niche? If yes, what roles are organoids playing inside stem cell niches? These questions have occupied the minds of stem cell scientists for a long time. Brain [2-4], heart [5,6], intestine $[7,8]$, skeletal muscle [9], stem cells have been identified and shown to play the functional role of tissue replacement and sustenance of specific tissue integrity [10-12] thereby providing tools for drug discovery and for studies on regenerative medicine [13]. For example, hope has been raised in the potential of stem cells [14] for use in therapy see reviews by [15-18]. Proposed that stem cells are progenitor [19] cells that (in appropriate medium) indefinitely self-renew and differentiate thereby causing more specialized cells and tissue to be produced. These investigators have studied stem cells using methods involving morphology [19], marker expression as observed by flow cytometry and fluorescence analysis, real time polymerase chain reaction, and proliferation and differentiation [18,20-22].

\section{Organoid/Stem Cell Activities in Niche Micro- environment}

Earlier reports showed that neural stem cell microenvironment can become so complex making neurogenesis to be associated with stem cells transformation to radial glial cells and that the complexity of this microenvironment increases due to the presence of neuronal progenitors, differentiated cells and extracellular signaling molecules see review by

* Corresponding author: Uche A Osunkwo, Department of Pharmacology \& Therapeutics, University of Abuja, Abuja, Nigeria, Tel: +234-803-329-6137

Accepted: April 22, 2021

Published online: April 24, 2021

Citation: Osunkwo UA, Ezeunala MN, Okolo RU, et al. (2021) Specificity of Organoids for Precision Tissue Regeneration: A Review. Insights Stem Cell Res Ther 3(1):23-30 
$[23,24]$. It has also been reported that cord blood-borne fibroblasts are capable of independent re-assembly and formation of hematopoietic stem cell niche [25-27]. Stem cell niche is reported to be associated with quiescence to enable lifelong tissue maintenance and premature exhaustion protection see review by [28] and that mechanical forces operating in the niche center on transcription factors as well as chromatin remodeling enzymes engaging in gene expression see review by [29]. Stem cell microenvironment could therefore be a localized portion where cell identification for sorting based on similarity, re-assembly, transformation into pre-organoids, organoidogenesis [12] and differentiation into new tissues takes place [30,21].

Signaling within stem cell environment has been known to be relevant to their proliferation, differentiation and sustenance of these activities including process of transcription and role of transcription factors [31-35]. In their review on mechanisms of stem cell self-renewal, [36] stated that cell-extrinsic signals from the niche microenvironment controlled cell-intrinsic mechanisms thereby maintaining and controlling stem cell functions in tissues. Therefore, it is imaginable that the signaling relationship between stem cells in the niche and those of stem cells in tissues remain sustained. Hematopoietic stem cells [26], for example, have been described to be present in an anatomical location described as the hematopoietic stem cell niche see review by [37]. Signaling molecules have been identified for neural stem cells within a microenvironment and such signals as retinoic acid and fibroblast growth factors [38] are included. In a culture medium containing Activin A, mouse epiblast stem cells caused self-renewal of epiblast stem cells through direct activation of Nanog (a transcription factor) [39].

For organoids, the possibility of their satisfying the expectation of specific tissue regeneration has been reviewed $[40,41]$ for drug discovery and disease modeling [41-46]. Organoids of various stem cell types possessing the capability of memory retention of the genetic composition of their organs $[10,47,48]$ have been identified and proposed to be potentially useful in serving as a template for in-situ tissue regeneration $[11,16,41,46,48]$ and for experimental purposes [41]. Organoids also ensure genomic stability $[49,50]$. Organoids have been made from stem cells for many organs see editorial by [45]. These in-vitro generated 3D self-assembly of stem cells which possess the three germ layer components [51] have been described for the hematopoietic and endothelial system $[26,27,52]$, cardiomyocytes [5,6], human somatic cells $[53]$, intestine $[7,8,10]$ and brain $[2-4,11]$. Specifically, organoids of human origin have been generated and reported for kidney [54], stomach [55,56], intestine [57], lung [58], brain $[11,59]$, and eyes [60] and these organoids have all shown the major characteristics of organoids including genetic similarity [61] and morphological resemblance of their parent organ [61]. Organoids produced from skeletal myoblasts, reversibly secreted bioactive compounds and have been suggested to be an improved method of muscle gene therapy [30]. Various translational applications of organoids derived from adult stem cells have been reviewed [41,62]. Also, stem cell-derived self-organizing three-dimensional organoids are thought to have a significant place in respiratory diseases research and development [63]. Finally, from their review of single-cell transcriptomics on organoids, [64] have suggested that a merging of organoid and single-cell genomics studies will augur well for precision medicine of tomorrow. Therefore, application of organoid culture technology and development to studies on cancer pathology and conventional pharmacological studies $[17,65,66]$ will also augur well for development of precision tissue regeneration.

\section{Organoidogenesis and Signaling Pathways}

The mechanisms for elaboration of organoids and their inherent memory (recapitulation) of the genetic composition of their organs $[67,68]$ from within stem cell niches such as the hematopoietic stem cell niche $[22,37]$ or the spermatogonial stem cell see (Figure 1) $[14,19]$ may be determined and explainable by recalling the process of transcription and how transcription factors participate in development of stem cell pluripotency. In the opinion of [51], presenting growth factors and signals to pluripotent stem cells creates opportunity for differentiation and morphogenesis as well as consequent organoidogenesis. These investigators also maintained that Wnt, FGF and retinoic acid (RA) are some of the factors that support patterning and morphogenesis [51] in stem cell development. In a report based on their study of Wnt/b-Catenin signaling during stem cell proliferation in Murine intestine, [69] pointed out that Wnt/b-catenin signaling and stem cell proliferation caused an increase [69] following depletion of cystic fibrosis transmembrane conductance regulator (Cftr). Again, starting with human embryonic stem cell derived intestinal organoids possessing gene expression patterns that generated human duodenum or ileum depicting regional specification of the developing human intestine [67], these authors showed that active FGF and WNT signaling was associated with organoids which possessed gene expression patterns that generated human duodenum or ileum suggesting regional specification of the developing human intestine [67]. By analyzing regulatory networks of murine small intestinal organoids, [12] suggested that multifactorial mechanisms may be involved in genetic rearrangement which gives rise to inflammatory bowel disease.

In another study, [70] reported that receptor-interacting protein kinase 3 was strongly associated with cellular mechanisms for reprogramming mouse embryonic fibroblast cells into induced pluripotent stem cells elaboration making these investigators to suggest that receptor-interacting protein kinase 3 removal was associated with markedly reduced reprogramming of induced pluripotent stem cells [70]. It is apparently suggested from here that in the presence of necroptosis kinase (receptor-interacting protein kinase 3 ), both programmed cell death and induction of reprogramming of pluripotent stem cells take place (possibly simultaneously). A specific study that relates transport nucleic acids to cellular pathobiology can be found in [12] who reported that by profiling $m R N A$, microRNA and other RNA expression, they were able to observe that differential differentiation of Paneth cells and goblet cells contributed to aetiology of inflammatory bowel disease. 


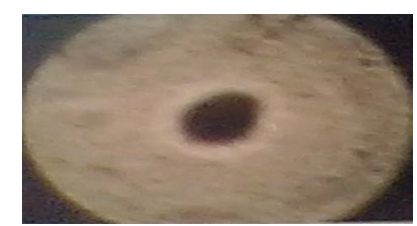

Day 0: Single spermatogonial stem cell (SSC).

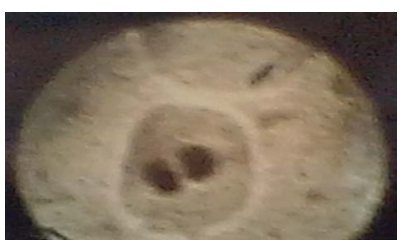

Day 3-5: Pre-Proliferation SSCs

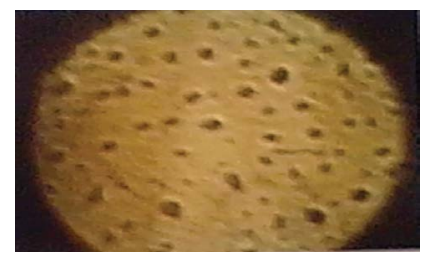

Day 5: Proliferated SSCs. (3 steps of proliferation).

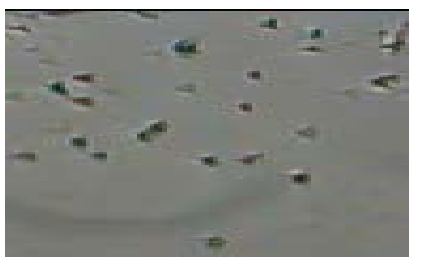

Day 7: Pre-organoids (post prompt for organoidogenesis. Cells are no longer spheroid).

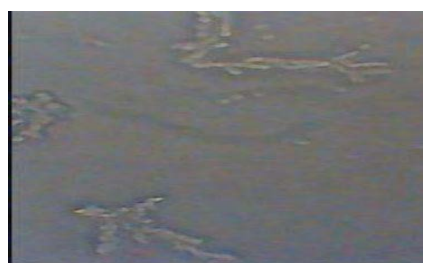

Day 15: Organoids in culture (prompted with retinol, 2 steps of organoidogenesis).

Figure 1: Illustrating position of organoids in organoidogenesis.

Photomicrographs showing developmental days 0-5 were prepared using locally adapted Mezo stain $[19,14]$ while those for days above 5 were prepared using Evans Blue (Standard Fluka). Microscope eye piece was interfaced with a camera and computer output for higher than normal magnification.

The point of emphasis here is the role of identified signaling process that initiates and/or supports stem cell proliferation. Recall that stem cell proliferation precedes organoidogenesis $[10,11,22]$ because organoids are derived from gene-signaling-directed self-assembly of like-stem cells. In a study on the role of several signaling factors in niche-regulated stem cell renewal to give rise to elaboration of organoids (enteroids from small intestine and colonoids from the colon), these investigators described an in vitro organ-like culture system for growth and maintenance of human enteroids and colonoids from the gastrointestinal tract [10]. In another report, mass cytometry has been used for multiplexed single-cell analysis of post-translational modification signaling networks and the results showed the presence of cell-type and cell-state specific signaling networks in stem cells during development of intestinal organoids [71].

In liver organoids, paracrine signals of mesoderm origin have been reported to support maturation of the hepatocyte $[72,73]$ have also reported hepatobiliary organogenesis in which in-vitro elaborated hepatobiliary organoids recapitulated and expressed structural and functional properties of hepatic and biliary organs. They further stated that their laboratory method did not involve use of exogenous cells or genetic manipulation [73]. In a study of the causal relationship between hematopoietic progenitors and erythropoiesis in perivascular niche of bone marrow, [22] reported that one of the reasons for the observation of LepR+ cells being one hundred fold more frequent than hematopoietic stem cells was the requirement of LepR+ derived stem cell factor for maintenance of c-kit+-restricted hematopoietic progenitors [22]. 
These investigators further reported the possibility of cellular specialization within the perivascular niche where endothelial cell stem cell factor was mostly required for production of hematopoietic stem cells [22]. Again, reversible loss of signaling pathway secondary to age-related failure of regenerative potential of mouse skeletal muscle fibro-adipogenic progenitors has been reported [74]. These authors suggest that transplantation of fibro-adipogenic progenitors sourced from young animals reversed the pathology and could serve as a treatment modality for purposes of myogenic rejuvenation.

Differentiation of human embryonic stem cells (hESCs) into thalamic organoids has been reported [75]. These investigators also reported that RNA sequencing of single-cells showed that thalamic organoids recapitulated development of the thalamus. Similarly, it has been reported that organoid system caused the differentiation of human pluripotent stem cells into $T$ cells, a process that is highly relevant to autologous $T$ cell immunotherapy [76]. These researchers further reported that their methodology was efficient for production of functional, mature $\mathrm{T}$ cells from human pluripotent stem cells.

\section{Biochemical Mechanisms in Organoid Speci- ficity}

It is true that natural molecular biology mechanisms of protein synthesis and macromolecule designing and fabrication are also part of tissue repair process. [34] in an early review article have observed that microRNA pathway components in nuage (a germline organelle) are necessarily required for MicroRNAs (thought to regulate post-trascriptional gene expression) regulatory function. However, using organoids in experimental tissue repair studies provides an opportunity for further understanding of the "central dogma of molecular biology". In the review on organoid engineering arising from stem cells, [77] proposed that in vitro re-creation of processes involved in human morphogenesis can lead to creation of tissue-like structure formation [77]. Similarly, [78] in their own review have suggested that biochemical and biophysical properties of engineered matrices are adjustable to supporting development and maturation of organoid cultures. In vitro generated skeletal muscle organoids have long been suggested to be a possibility for use as gene therapy and this suggestion arose from the observation of reversibility of the effects of experimentation in gene therapy [30]. Nicotinamide riboside (NAD+ potentiating agent) has been reported to decrease mitochondrial activity by increasing its clearance thereby markedly increasing hematopoietic stem cell progenitor pool [79] and these investigators suggested the potential use of nicotinamide riboside in management of hematological failure secondary to chemotherapy or radiotherapy [80].

Lewis, et al. [80] have reported that organoids retained the biological clock of the cell and is measurable using epigenetic clock (biomarker). The possibility of organoids deploying innate biochemical mechanisms for achieving this observed retention exists. These investigators reported that DNA methylation patterns manifested gastrointestinal segment-related differences and that stem cell enriched organoids from the small intestine and those from the colon deferentially ex- pressed epigenetic age [80]. Here, we find that the earlier reports on possible biochemical mechanisms (especially signaling) of organoid functionality holds $[51,67,69]$.

\section{Organoids and Tissue Regeneration}

In-situ tissue regeneration or tissue transplantation technology has become necessary because of high demand for replacement. For this and other reasons, organoid formation from stem cell self-reorganization has recently become topical and reports are beginning to emerge on advancement of this technology. Small cystic organoids have been used for the generation of in vitro macroscopic intestinal tubes [81]. These investigators started with cultured mouse intestinal stem cells that gave rise to epithelial organoids which selfaligned and fused to form macroscopic hollow structures reminiscent of intestinal tubes [81]. Again, patient-derived neurons and organoids have been suggested to be useful in drug testing and in comprehension of pathogenesis of Parkinson's disease [82]. Similarly, starting with human embryonic stem cell-derived intestinal organoids, [67] showed that active FGF and WNT signaling was associated with organoids possessing gene expression patterns that generated human duodenum or ileum depicting regional specification of the developing human intestine [67]. Likewise, [21] have reported that epithelial cells originating from the lung could be grown in appropriate culture medium to result in organoids and this process can be done for human or mouse lungs.

Mouse mammary organoids derived from basal mammary epithelial cells were capable of milk-production and could be used for research purposes [83]. In the report of [30], organoids produced from skeletal myoblasts secreted marked levels of human growth hormone when subcutaneously administered and this result led these investigators to suggest that the organoid production and administration process was an advantageous form of gene therapy for skeletal muscle [30]. In their report of literature review, [84] stated that adult stem cell organoids derived from healthy or diseased epithelium of many organs obtained from malignant, hereditary and infectious diseases patients have been useful in drug development studies which may be beneficial towards development of personalized and regenerative medicine.

Putting to question the reproducibility of cortical organoid generation, [85] elaborated cortical organoids from many stem cell lines for more than one hundred days and concluded that the consistency and reliability of their methodology would be required for future production for disease modeling and massive differentiation studies [85]. Brain organoids from stem cells and their relevance in advancement of neurobiology [74], and the generation of intestinal organoids [8,86] have been reviewed.

Temporal relationships have been identified during organoid elaboration and fate determination. For example, in a study of the transformation from endoderm through hindgut spheroids to mature organoids, [87] reported that $13 \%$ of observable spheroids turned out to be pre-organoids which later matured to become intestinal organoids. These authors further reported a variation in cell number, diameter and morphology of spheroids. In considering diameter 
and morphology of spheroid inner mass, they concluded that spheroid diameter and inner mass are enriching factors for intestinal spheroid maturation [87]. Again, it has been shown that a time-dependent effect manifests when human embryonic stem cell-derived intestinal organoids are in contact with active FGF and WNT and a $\beta$-catenin stabilizer signaling, and that duration of exposure to the signaling process determines whether human duodenum-like or ileum-like producing organoids are elaborated [67].

The possibility of formulating organoids and or stem cellular materials including biomaterials $[47,88,89]$ and cytoceuticals see review by [90] into treatment regimens is gradually becoming realistic. Cytoceuticals derived from biological, non-biological or a combination of both may become readily available and possibly abundant in this decade subject to positive efficacy results from clinical trials. Exciting potentials of organoid technology and its applications in bench and clinical settings have been extensively reviewed by [62]. Other reviews $[30,41,47,89]$ also agree that various potential clinical applications of organoid technology was worth the effort in laboratory development. In a review on the possibility of developing salivary gland organoids from stem cells for the treatment of glandular hypo-function and xerostomia (dry mouth syndrome) [89] pointed out that a possible strategy lies in transplantation of autologous epithelial stem and progenitor cells of salivary gland origin. Similarly, human $\mathrm{T}$ cell differentiation (as in thymopoiesis) has been suggested to be a strong potential in the search for stem-cell-based engineered T cell therapies [91]. Again, the possibility of organ fabrication using synthetic or natural biomaterials especially a person's body cells as an intervention strategy to stop-gap supply and demand differences in organ transplants for the future has been suggested [88].

Now, these possibilities in cellular genetic and macromolecular rearrangements leading from stem cells to organoids for the purposes of effecting tissue regeneration and correcting disease-related degeneration can be reviewed from the viewpoint of cellular and tissue mis-arrangement that gives rise to disease. For example, [92] studied gastrointestinal organoids generation from crypt stem cells of healthy controls and those of patients with celiac disease and reported distinct dissimilarities in the phenotypes of organoids as well as significant variations in genetic material expression between these two groups. These investigators concluded that a differential gene expression during epithelial-mesenchymal transition existed among the two study groups and that this differential organoid gene expression might be responsible for the deranged crypt and villus axis in the pathogenesis of celiac disease [92]. Organoids of liver origin made from induced pluripotent stem cells for multiple species are now available for experimental medicine research [93]. Following a review of several literature materials, the possibility has been suggested that optimized treatment strategies may be targeted for an individual patient [94] using organoids. Using organoid CRISPR/Cas9 (a defense mechanism of bacteria) precision genome editing technology, [68] as well as [84] have stated the possibility of accurately integrating exogenous DNA sequences at gene positions chosen by the investigator. Therefore, gene therapy in the near future will be boosted by such gene manipulation technologies. The report by [95] has characterized oral mucosa organoid as being useful in prevention, diagnoses, potential treatment of cancer and in pharmacological screening processes [95]. These recent reports when put together clarify the establishment of a new human disease and treatment model system.

\section{Statement on Conflict of Interest by the Re- search Team}

Nothing to declare.

\section{Acknowledgment}

We hereby acknowledge the support of Nigerian government tertiary education trust fund (TETFUND) Institution (University of Abuja, Abuja) based research grant No. 6 of 2015 and the National Institute for Pharmaceutical Research and Development (NIPRD) pipeline projects research grant (2020) awarded to Principal Investigator, Prof. Uche A. Osunkwo.

\section{References}

1. Regent F, Chen HY, Kelley RA, et al. (2020) A simple and efficient method for generating human retinal organoids. Mol Vis 26: 97105.

2. Pasca SP (2018) The rise of three-dimensional human brain cultures. Nature 553: 437-445.

3. Mansour AA, Goncalves JT, Bloyd CW, et al. (2018) An in vivo model of functional and vascularized human brain organoids. Nature Biotechnology 36: 432-441.

4. Xuyu Q, Song H, Ming G-L (2019) Brain organoids: Advances, applications and challenges. Development 146: dev166074.

5. Chan KK, Zhang J, Chia NY, et al. (2009) KLF4 and PBX1 directly regulate NANOG expression in human embryonic stem cells. Stem Cells 27: 2114-2125.

6. Elisa G, Bellin M, Sala L, et al. (2017) Three-dimensional cardiac microtissues composed of cardiomyocytes and endothelial cells co-differentiated from human pluripotent stem cells. Development 144: 1008-1017.

7. Munera JO, Sundaram N, Rankin SA, et al. (2017) Differentiation of human pluripotent stem cells into colonic organoids via transient activation of BMP signaling. Cell Stem Cell 21: 51-64.

8. Daichi O, Yamashita M, Nakanishi A, et al. (2018) Generation of intestinal organoids suitable for pharmacokinetic studies from human induced pluripotent stem cells. Drug Metab Dispos 46: 1572-1580.

9. Ebert AD, Yu J, Rose FF, et al. (2009) Generation and differentiation of induced pluripotent stem cells from a spinal muscular atrophy patient. Nature 457: 27780.

10. Mahe MM, Sundaram N, Watson CL, et al. (2015) Establishment of Human Epithelial Enteroids and Colonoids from Whole Tissue and Biopsy. J Vis Exp 6: 52483.

11. Bonsang K, Choi B, Yoon K-J, et al. (2019) Past, present, and future of brain organoid technology. Mol Cells 42: 617-627.

12. Treveil A, Sudhakar P, Matthews ZJ, et al. (2020) Regulatory network analysis of paneth cell and goblet cell enriched gut organoids using transcriptomics approaches. Mol Omics 16: 39-58. 
Citation: Osunkwo UA, Ezeunala MN, Okolo RU, et al. (2021) Specificity of Organoids for Precision Tissue Regeneration: A Review. Insights Stem Cell Res Ther 3(1):23-30

13. Feng W, Jie K, Yi-Yao C, et al. (2018) Is human-induced pluripotent stem cell the best optimal? Chin Med J 131: 852-856.

14. Osunkwo UA, Ezeunala, Mercy N, et al. (2011) Optical microscope gross characterization of rat neonatal testicular germline stem cell expansion in vitro. International Journal of Genetics and Molecular Biology 3: 39-44.

15. Daley GQ, Scadden DT (2008) Prospects for stem cell-based therapy. Cell 132: 544-548.

16. Rodolfa KT (2008) Inducing pluripotency. Stem Book. (ed). The Stem Cell Research Community, Stem Book.

17. Cosset E, Locatelli M, Marteyn A, et al. (2019) Human neural organoids for studying brain cancer and neurodegenerative diseases. J Vis Exp 148.

18. Gargiulo C, Pham VH, Hai NT, et al. (2015) Isolation and characterization of multipotent and pluripotent stem cells from human peripheral blood. Stem Cell Discovery 5: 19-32.

19. Osunkwo UA, N Ezeunala Mercy, MO Duru Osunkwo, et al. (2009) A comparative study of the gross structure and distribution of spermatogonia-like cells in the testis of pre-pubertal and mature adult rats. Journal of Cell and Animal Biology 3: 202-207.

20. Shoichi D, Sato T (2015) Mini-gut organoids: Reconstitution of the stem cell niche. Annu Rev Cell Dev Biol 31: 269-289.

21. Barkauskas Christina E, Mei-I Chung, Fioret B, et al. (2017) Lung organoids: Current uses and future promise. Development 144: 986-997.

22. Comazzetto MS, Murphy M, Berto S, et al. (2019) Restricted hematopoietic progenitors and erythropoiesis require scf from leptin receptor+ niche cells in the bone marrow. Cell Stem Cell 24: 477-486.

23. Kazanis I, Lathia J, Moss L, et al. (2008) The neural stem cell microenvironment. Stem Book, (ed). The Stem Cell Research Community, Stem Book.

24. Murrow LM, Weber RJ, Gartner ZJ (2017) Dissecting the stem cell niche with organoid models: An engineering-based approach. Development 144: 998-1007.

25. Martinez-AgostoJulian A, Hanna KA Mikkola, Hartenstein V, et al. (2007) The hematopoietic stem cell and its niche: A comparative view. Genes Dev 21: 3044-3060.

26. Jonathan H, Kfoury Y, Scadden DT (2016) Hematopoietic stem cell niche in health and disease. Annu Rev Pathol 11: 555-581.

27. Alice $P$, Sacchetti B, Corsi A, et al. (2017) Human umbilical cord blood-borne fibroblasts contain marrow niche precursors that form a bone marrow organoid in vivo. Development 144: 10351044.

28. Arai F, Suda T (2008) Quiescent stem cells in the niche. Stem Book, (ed). The Stem Cell Research Community, Stem Book.

29. Chen $X, X u H$, Yuan $P$, et al. (2008) Integration of external signaling pathways with the core transcriptional network in embryonic stem cells. Cell 133: 1106-1117.

30. Herman V, Tatfo M, Shansky J, et al. (1996) Tissue-engineered skeletal muscle organoids gene therapy for reversible gene therapy. Hum Gene Ther 7: 2195-2200.

31. Hay DC, Sutherland L, Clark J, et al. (2004) Oct-4 knockdown induces similar patterns of endoderm and trophoblast differentiation markers in human and mouse embryonic stem cells. Stem Cells 22: 225-235.
32. Camus A, Perea-Gomez A, Moreau A, et al. (2006) Absence of nodal signaling promotes precocious neural differentiation in the mouse embryo. Dev Biol 295: 743-755.

33. Ivanova N, Dobrin R, Lu R, et al. (2006) Dissecting self-renewal in stem cells with RNA interference. Nature 442: 533-538.

34. Reynolds S, Ruohola-Baker H (2008) MicroRNA's role in germline differentiation. The Stem Cell Research Community, Stem Book.

35. Chan KK, Zhang J, Chia NY, et al. (2009) KLF4 and PBX1 directly regulate NANOG expression in human embryonic stem cells. Stem Cells 27: 2114-2125.

36. Shenghui H, Nakada D, Morrison SJ (2009) Mechanisms of stem cell self-renewal. Annu Rev Cell Dev Biol 25: 377-406.

37. Purton LE, Scadden DT (2008) The hematopoietic stem cell niche. The Stem Cell Research Community, Stem Book.

38. Kazanis I, Lathia J, Moss L, et al. (2008) The neural stem cell microenvironment. The Stem Cell Research Community, Stem Book.

39. Boris G, Wu G, Bernemann C, et al. (2010) Conserved and divergent roles of FGF signaling in mouse epiblast stem cells and human embryonic stem cells. Cell Stem Cell 6: 215-226.

40. Meritxell H, Knoblich JA, Lutolf MP, et al. (2017) The hope and the hype of organoid research. Development 144: 938-941.

41. Jarno D, Clevers H (2017) Translational applications of adult stem cell-derived organoids. Development 144: 968-975.

42. Guguen-Guillouzo C, Corlu A, Guillouzo A (2010) Stem cell-derived hepatocytes and their use in toxicology. Toxicology 270: 3-9.

43. Liu W, Deng Y, Liu Y, et al. (2013) Stem cell models for drug discovery and toxicology studies. J Biochem Mol Toxicol 27: 17-27.

44. Csobonyeiova M, Polák S, Danisovic L (2016) Toxicity testing and drug screening using iPSC-derived hepatocytes, cardiomyocytes, and neural cells. Can J Physiol Pharmacol 94: 687-694.

45. Little Melissa H (2017) Organoids: A special issue. Development 144: 935-937.

46. Xuan HB, Pek NMQ, Soh B-S (2018) Disease modeling using 3D organoids derived from human induced pluripotent stem cells. Int J Mol Sci 19: 936-953.

47. Ninouk A, Defize LHK (2017) Dawn of the organoid era: 3D tissue and organ cultures revolutionize the study of development, disease, and regeneration. Bioessays.

48. Ruth L, Leeb CM, Shugartc EC, et al. (2019) Human organoids: A new dimension in cell biology. Mol Biol Cell 30: 1129-1137.

49. Clevers H (2016) Modeling development and disease with organoids. Cell 165: 1586-1597.

50. Rossi G, Manfrin A, Lutolf MP (2018) Progress and potential in organoid research. Nat Rev Genet 19: 671-687.

51. McCauley Heather A, Wells JM (2017) Pluripotent stem cell-derived organoids: Using principles of developmental biology to grow human tissues in a dish. Development 144: 958-962.

52. Choi KD, Yu J, Smuga-Otto K, et al. (2009) Hematopoietic and endothelial differentiation of human induced pluripotent stem cells. Stem Cells 27: 559-567.

53. Yu J, Vodyanik M, Smuga-Otto K, et al. (2007) Induced pluripotent stem cell lines derived from human somatic cells. Science 318: 1917-1920. 
Citation: Osunkwo UA, Ezeunala MN, Okolo RU, et al. (2021) Specificity of Organoids for Precision Tissue Regeneration: A Review. Insights Stem Cell Res Ther 3(1):23-30

54. Takasato M, Er PX, Becroft M, et al. (2014) Directing human embryonic stem cell differentiation towards a renal lineage generates a self-organizing kidney. Nat Cell Biol 16: 118-126.

55. McCracken KW, Catá EM, Crawford CM, et al. (2014) Modelling human development and disease in pluripotent stem-cell derived gastric organoids. Nature 516: 400-404.

56. McCracken KW, Aihara E, Martin B, et al. (2017) Wnt/B-catenin promotes gastric fundus specification in mice and humans. Nature 541: 182-187.

57. Spence JR, Mayhew CN, Rankin SA, et al. (2011) Directed differentiation of human pluripotent stem cells into intestinal tissue in vitro. Nature 470: 105-109.

58. Dye BR, Hill DR, Ferguson MAH, et al. (2015) In vitro generation of human pluripotent stem cell derived lung organoids. Elife 4: e05098.

59. Lancaster MA, Renner M, Martin CA, et al. (2013) Cerebral organoids model human brain development and microcephaly. Nature 501: 373-379.

60. Nakano T, Ando S, Takata N, et al. (2012) Self-formation of optic cups and storable stratified neural retina from human ESCs. Cell Stem Cell 10: 771-785.

61. Valentina B, Destrade M, Goriely A (2020) Mechanics of human brain organoids. Phys Rev E 101: 022403.

62. Takahashi T (2018) Organoids for drug discovery and personalized medicine. Annual Review of Pharmacology and Toxicology 59: 447-462.

63. Li Y, Wu Q, Sun X, et al. (2020) Organoids as a powerful model for respiratory diseases. Stem Cells Int.

64. Agnieska B, Treutlein B, Gray Camp J (2019) High-throughput single-cell transcriptomics on organoids. Curr Opin Biotechnol 55: 167-171.

65. Hanxiao X, Lyu X, Yi M, et al. (2018) Organoid technology and applications in cancer research. J Hematol Oncol 11: 116.

66. Hanxiao X, Jiao Y, Qin S, et al. (2018) Organoid technology in disease modelling, drug development, personalized treatment and regeneration medicine. Exp Hematol Oncol 7: 30.

67. Yu-Hwai T, Nattiv R, Dedhia PH, et al. (2017) In vitro patterning of pluripotent stem cell-derived intestine recapitulates in vivo human development. Development 144: 1045-1055.

68. Driehuis E, Clevers H (2017) CRISPR/Cas 9 genome editing and its applications in organoids. Am J Physiol Gastrointest Liver Physiol 312: G257-G265.

69. Strubberg Ashlee M, Liu J, Walker NM, et al. (2018) Cftr modulates wnt/b-catenin signaling and stem cell proliferation in murine intestine. Cell Mol Gastroenterol Hepatol 5: 253-271.

70. Ahmad A-M, Tian B, Efstathiou NE, et al. (2019) Receptor interacting protein kinase 3 (RIP3) regulates iPSCs generation through modulating cell cycle progression genes. Stem Cell Research 35: 101387.

71. Xiao Q, Sufi J, Vlckova P, et al. (2019) Single-cell signalling analysis of heterocellular organoids. bioRxiv 5: 1-7.

72. Akihiro A, Aihara E, Watson C, et al. (2017) Paracrine signals regulate human liver organoid maturation from induced pluripotent stem cells. Development 144: 1056-1064.

73. Fenfang W, Wu D, Ren Y, et al. (2019) Generation of hepatobiliary organoids from human induced pluripotent stem cells. J Hepatol 70: 1145-1158.
74. Laura L, Karaz S, Stuelsatz P, et al. (2019) Aging disrupts muscle stem cell function by impairing matricellular wisp1 secretion from fibro-adipogenic progenitors. Cell Stem Cell 24: 433-446.

75. Yangfei X, Tanaka Y, Cakir B, et al. (2019) HESC-derived thalamic organoids form reciprocal projections when fused with cortical organoids. Cell Stem Cell 24: 487-497.

76. Amelie M-H, Seet CS, Li S, et al. (2019) organoid-induced differentiation of conventional $t$ cells from human pluripotent stem cells. Cell Stem Cell 24: 376-389.

77. Silva Teresa P, Cotovio JP, Bekman E, et al. (2019) Design principles for pluripotent stem cell-derived organoid engineering. Engineering Cell Systems.

78. Kratochvil Michael J, Seymour AJ, Li TL, et al. (2019) Engineered materials for organoid systems. Nature Reviews Materials 4: 606-622.

79. Nicola V, Campos V, Girotra M, et al. (2019) The NAD-booster nicotinamide riboside potently stimulates hematopoiesis through increased mitochondrial clearance. Cell Stem Cell 24: 405.e7-418.e7.

80. Lewis Sophia K, Nachun D, Martin MG, et al. (2020) DNA methylation analysis validates organoids as a viable model for studying human intestinal aging. Cell Mol Gastroenterol Hepatol 9: 527541.

81. Norman S, Tsukamoto Y, Kujala P, et al. (2017) Intestinal epithelial organoids fuse to form self-organizing tubes in floating collagen gels. Development 144: 1107-1112.

82. Nick M, Kim S, Krainc D (2020) Organoid and pluripotent stem cells in parkinson's disease modeling: An expert view on their value to drug discovery. Expert Opin Drug Discov 15: 427-441.

83. Jamieson Paul R, Dekkers JF, Rios AC, et al. (2017) Derivation of a robust mouse mammary organoid system for studying tissue dynamics. Development 144: 1065-1071.

84. Benedetta A, Hendriks D, Beumer J, et al. (2020) Fast and efficient generation of knock-in human organoids using homology-independent CRISPR/Cas9 precision genome editing. Nat Cell Biol 22: 321-331.

85. Se-Jin Y, Elahi LS, Pașca AM, et al. (2019) Reliability of human cortical organoid generation. Nat Methods 16: 75-78.

86. Nina K, Pratscher B, Meneses AMC, et al. (2020) Generation of differentiating and long-living intestinal organoids reflecting the cellular diversity of canine intestine. Cells 9: 822.

87. Natasha A, Alsous JI, Guggenheim JW, et al. (2017) A process engineering approach to increase organoid yield. Development 144: 1128-1136.

88. Piyush B, Schweller RM, Khademhosseini A, et al. (2014) 3D Biofabrication strategies for tissue engineering and regenerative medicine. Annu Rev Biomed Eng 16: 247-276.

89. Isabelle L, Movahednia MM, Adine C, et al. (2017) Salivary gland regeneration: Therapeutic approaches from stem cells to tissue organoids. Stem Cells 35: 97-105.

90. Osunkwo UA, Okolo RU, Bassi PU, et al. (2020) Stem cell dynamics and cytoceuticals for therapeutics and toxicity screening: A review. Insights Stem Cell Res Ther 2: 15-22.

91. Seet Christopher S, He C, Bethune MT, et al. (2017) Generation of mature T cells from human hematopoietic stem and progenitor cells in artificial thymic organoids. Nat Methods 14: 521-530. 
92. Walburga D, Neurath MF, Zopf Y (2020) Intestinal ex vivo organoid culture reveals altered programmed crypt stem cells in patients with celiac disease. Sci Rep 10: 3535.

93. Nicole P, Inacio P, Huch M (2019) Liver organoids: From basic research to therapeutic applications. Gut 68: 2228-2237.
94. Xu H, Jiao Y, Qin S, et al. (2018) Organoid technology in disease modelling, drug development, personalized treatment and regeneration medicine. Exp Hematol Oncol 7: 30.

95. Dreihuis Else (2019) Organoids as a tool for fundamental and translational oncology research. Can organoids guide clinical decision making? 